\title{
Dictyostelium purpureum var. pseudosessile, a new variant of dictyostelid from tropical China
}

\author{
Pu Liu' ${ }^{1 \dagger}$, Yue Zou' ${ }^{1 \dagger}$, Jiangan Hou', Steven L. Stephenson ${ }^{2}$ and Yu Li ${ }^{1 *}$
}

\begin{abstract}
Background: Dictyostelid cellular slime molds (dictyostelids) are microscopic throughout their entire life cycle. The vegetative phase consists of single-celled amoeboid forms which live in the soil/leaf litter microhabitat of fields and forests along with animal dung, where they feed upon bacteria and other microbes, grow, and multiply until the available food supply is exhausted. When this happens, the amoeboid forms aggregate together in large numbers to form multi-celled pseudoplasmodia, which then give rise to fruiting bodies (sorocarps) that consist of supportive stalks and unwalled sori containing propagative spores.

Results: Dictyostelium purpureum var. pseudosessile, a new variant of dictyostelid, is described herein, based on morphological features and molecular data. This new variant was isolated from soil samples collected in two tropical areas of China. The complete spore-to-spore life cycle of this species, which required $50 \mathrm{~h}$, including spore germination, myxamoebae, cell aggregation, pseudoplasmodium, and sorocarp formation, was documented. Descriptions and illustrations are provided for this species based on our collections. Data from ontogeny, morphology and phylogeny analyses (SSU) of D. purpureum var. pseudosessile confirm that it is a Group 4 species according to the newly proposed classification of dictyostelids.
\end{abstract}

Conclusions: Our results suggest that the violet sori, widens at the midpoint of sorophore and simple recurved sorophore bases represent the prominent features for the new variant $D$. purpureum var. pseudosessile. The latter is a Group 4 species now known from two tropical areas of China where dictyostelids remains understudied.

Keywords: Dictyostelids, Taxonomy, Ontogeny, Phylogeny

\section{Background}

Dictyostelid cellular slime molds are key inhabitants of the soil and leaf litter layer of fields and forests, along with animal dung, where they feed mostly on bacteria [1-3]. Protists such as dictyostelids are important agents in bringing about quantitative changes to the bacterial microflora of soils and apparently play a role in maintaining the balance that exists between bacteria and other microbes in the soil $[4,5]$. Dictyostelids are members of Amoebozoa, a branch of eukaryotes separate from plants, fungi and animals. Their cells lack cell walls

\footnotetext{
* Correspondence: fungi966@126.com

${ }^{\dagger} \mathrm{Pu}$ Liu and Yue Zou contributed equally to this work.

${ }^{1}$ Engineering Research Center of Chinese Ministry of Education for Edible and Medicinal Fungi, Jilin Agricultural University, Changchun 130118,

People's Republic of China

Full list of author information is available at the end of the article
}

and resemble animal cells in organization, except for the presence of a contractile vacuole [6].

Since the first dictyostelid was described by Brefeld [7], more than 100 species have been reported and classified into one class, one order, two families and four genera [8]. These species are distinguished morphologically largely on the basis of differences in sorophore composition and branching pattern. However, a phylogenetic analysis based on $18 \mathrm{~S}$ ribosomal RNA (18S rRNA) and $\alpha$-tubulin indicated that the traditional morphological based classification traditionally used for these organisms did not hold up. The genera that had always been used for dictyostelids were not found to be monophyletic, and the various species were separated first into four groups [9] and then into eight groups [10], respectively. Furthermore, a new classification, based on unique $18 \mathrm{~S}$ rRNA sequence signatures, was proposed, and this 
provided an additional new insight into the taxonomy of the dictyostelids. As a result of this new classification, two families, nine genera and 92 new combinations were recognized at the level of species and variety [11].

The Hainan Province and Xishuangbanna region of Yunnan Province are both located south of Tropic of Cancer and are characterized by a tropical monsoon climate. However, studies of dictyostelids in this region are exceedingly limited and consist of only a single previous report of two species of dictyostelids from the Hainan Province [12]. To fill in this gap, focus of the present study was directed towards the morphology, ontogeny, and phylogeny of a new variant of dictyostelids recovered from soil samples collected at these two sites.

\section{Results}

After being processed, two isolates representing one new tropical variant of dictyostelids were yielded, with one isolate obtained from the Xishuangbanna Tropical Botanical Garden and a second isolate from the Qixianling Hot Springs National Forestry Park. It required $50 \mathrm{~h}$ for this species to complete its life cycle. Phylogenetic studies of the nuclear SSU rDNA showed that the new variant is a member of Group 4 (Fig. 1), based on the concepts of Schaap et al. [9], Romeralo et al. [10], and Sheikh et al. [11].

\section{Description of the new variant}

Dictyostelium purpureum var. pseudosessile Li Y., P. Liu, Y. Zou, S.L. Stephenson et J. Hou, sp. nov. (Fig. 2). MycoBank: MB825215.

\section{Holotype}

China. Hainan Province, Qixianling Hot Springs $\mathrm{Na}$ tional Forestry Park $\left(109^{\circ} 41^{\prime} \mathrm{E}\right.$ and $\left.18^{\circ} 42^{\prime} \mathrm{N}\right)$, located at an elevation of approximately $350 \mathrm{~m}$, from a soil sample collected within a broadleaf rainforest, 13 January 2015, HMJAU MR279 (4446).

\section{Diagnosis}

Sorocarps with several sessile droplets of water on one sorophore, gregarious, erect or semi-erect, unbranched, 4.3-7.3 mm high. Sorophore sinuous, white or violet, prostrate on the agar, widens at the midpoint of the

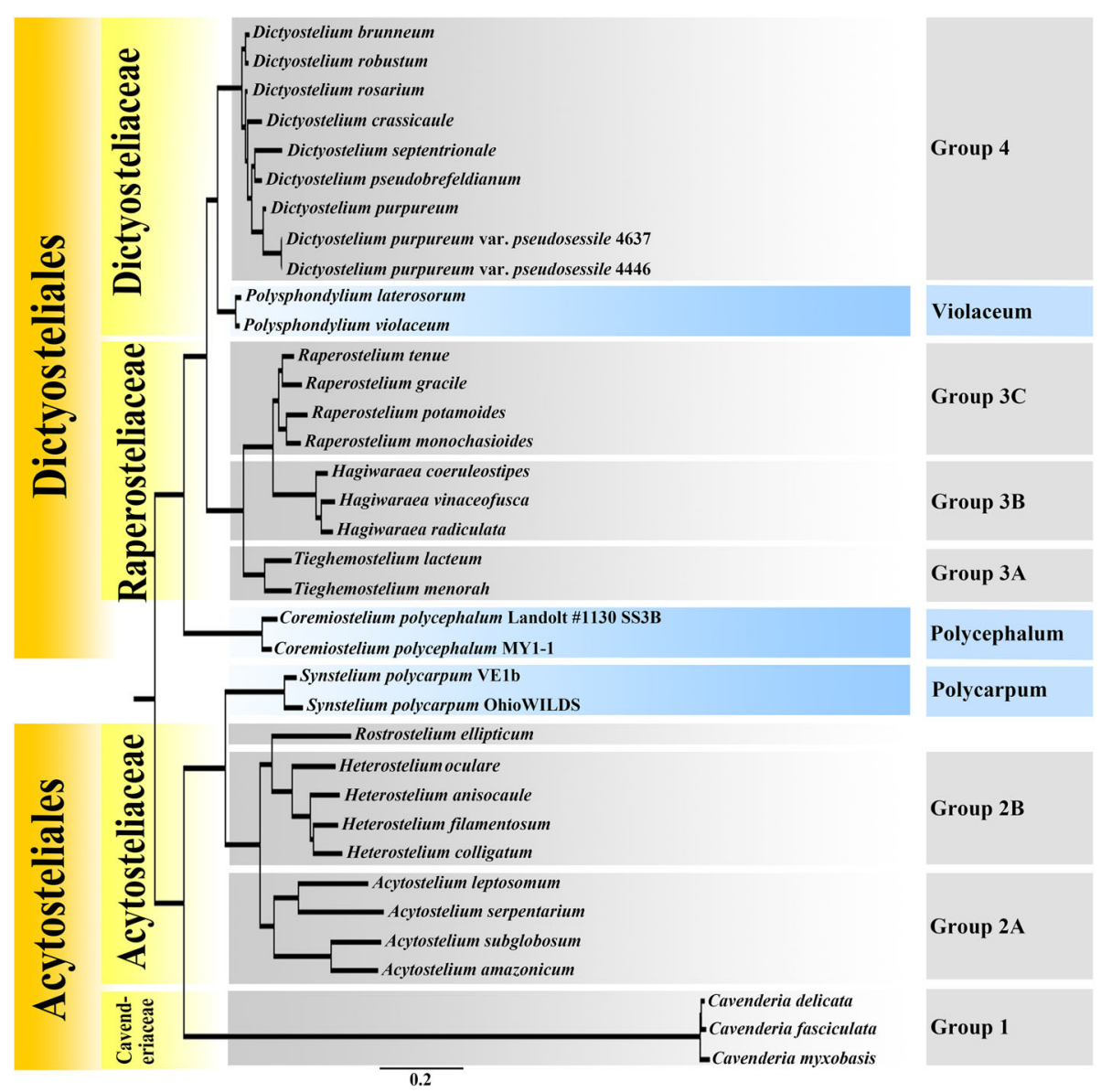

Fig. 1 Phylogenetic tree of dictyostelids based on SSU rRNA and portions of the SSU rRNA gene alignment, showing the molecular signatures of Dictyostelium purpureum var. pseudosessile 

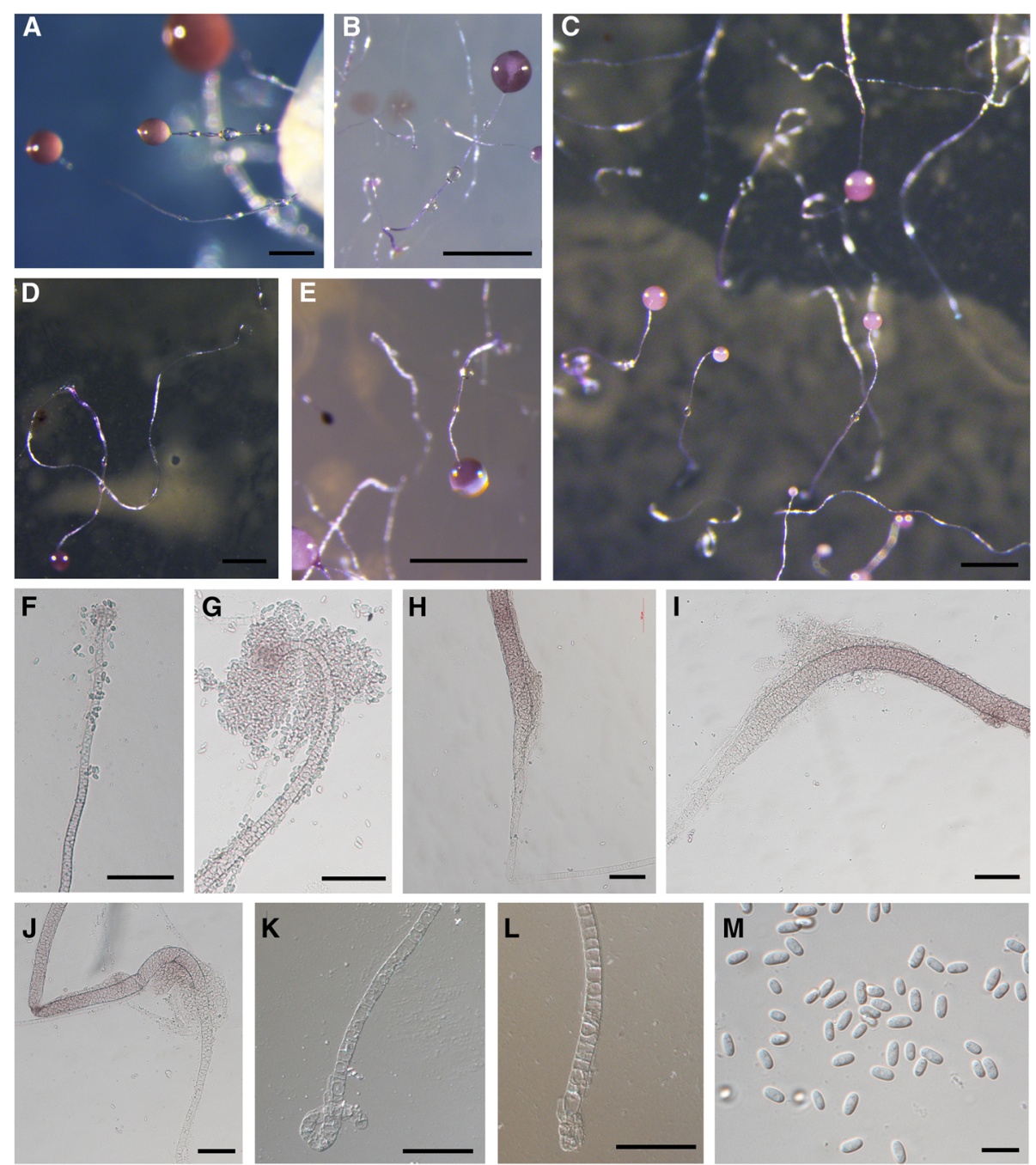

Fig. $\mathbf{2}$ Morphological features of Dictyostelium purpureum var. pseudosessile. a-e Sorocarps. $\mathbf{f} \mathbf{g}$ Sorophore tip. $\mathbf{h}-\mathbf{j}$ Mid-point of the sorophore. $\mathbf{k}, \mathbf{I}$ Sorophore base. $\mathbf{m}$ Spores. Scale bars: a: $200 \mu \mathrm{m}, \mathbf{b}, \mathbf{e}: 1 \mathrm{~mm}, \mathbf{c}: 0.5 \mathrm{~mm}, \mathbf{d}: 0.5 \mathrm{~mm}$; f-I: $20 \mu \mathrm{m}, \mathbf{m}: 10 \mu \mathrm{m}$

whole sorophore, bases recurve, tips capitate. Sori violet, globose. Spores oblong to elliptical, without obvious polar granules, $5.0-10 \times 2.5-5 \mu \mathrm{m}$. Aggregation streamed.

\section{Etymology}

From the Latin pseudosessile, referring to the several sessile droplets of water occurring on one sorophore.

\section{Description}

When cultured at $23 \mathrm{C}$ on non-nutrient agar with $E$. coli, sorocarps (Fig. 2-a-e) generally gregarious, erect or semi-erect, unbranched, commonly $4.3-7.3 \mathrm{~mm}$ high (average $5.7 \mathrm{~mm}$ ) with a variable number of sessile droplets of water occuring on one sorophore. Sorophore sinuous, white or violet, nearly half of the lower portion of the sorophore prostrate on the surface of the agar, the other upper portion of the sorophore erect or semi-erect on the agar. The erect or semi-erect portion of the sorophore widens (Fig. $2-\mathrm{h}-\mathrm{j}$ ) at the base and general consists of several tiers of cells, but the base (Fig. 2-k, l) of the prostrate portion of the sorophore recurves and usually consists of one or two tiers of cells $(10-37.5 \mu \mathrm{m}$ diam, average $18.5 \mu \mathrm{m}$ ). Tips (Fig. 2-f, g) capitate and consisting of one or two tiers of cells $(5-10 \mu \mathrm{m}$ diam, average $7.5 \mu \mathrm{m})$. Sori violet, globose to lemon-type, commonly $135-325 \mu \mathrm{m}$ diam (average $236 \mu \mathrm{m}$ ). Spores (Fig. $2-\mathrm{m}$ ) oblong to elliptical, $5.0-10 \times 2.5-5 \mu \mathrm{m}$, without obvious polar granules. Aggregations $(0.7-4 \mathrm{~mm})$ generally with asymmetrical streams. Early sorogens fusiform to coniform, migrate with stalk formation.

\section{Other specimens examined}

China. Yunnan Province, Chinese Academy of Sciences, Xishuangbanna Tropical Botanical Garden $\left(101^{\circ} 25^{\prime} \mathrm{E}\right.$ 
and $21^{\circ} 41^{\prime} \mathrm{N}$ ), located at an elevation of approximately $570 \mathrm{~m}$, from a soil sample collected from beneath $\mathrm{Hyd}$ nocarpus annamensis (Gagnep.) M. Lescot et Sleum, 20 January 2015, HMJAU MR273 (4637).

\section{Life cycle of $D$. purpureum var. pseudosessile}

Spores become larger when germination occurs; the latter begins with the appearance of a minute pore dissolved in the spore wall, and several myxamoebae are released from different spores in concert (Fig. 3-a). Myxamoebae are colorless, transparent, and irregular. A mass of myxamoebae aggregates to form a young aggregation center $32.5 \mathrm{~h}$ after the spores have been inoculated on agar (Fig. 3-b). Two hours later, the central portion of the aggregation begins to form a small central point with a prominent stream of cells (Fig. 3-c). After the aggregating of more and more myxamoebae, all myxamoebae aggregate at one central point, the prominent central point rises up and with fewer streams $35.5 \mathrm{~h}$ after the spores have been inoculated on agar (Fig. 3-d). One and a half hours later, the central cell aggregations rise up and begin to form pseudoplasmodia with indistinct sorophores (Fig. 3-e). Two and a half hours later, the sorophores begin to form (Fig. 3-f). Sorophores grow longer (Fig. $3-\mathrm{g}, \mathrm{h}$ ), which gives them the appearance of a fruiting sorophore (Fig. 3-i) at $42 \mathrm{~h}$, pseudoplasmodia form and undergo a very short period of movement. The sorophore is slightly curved, half of which is prostrate on the surface of the agar. After the formation of the sorophore, the sori begin to grow

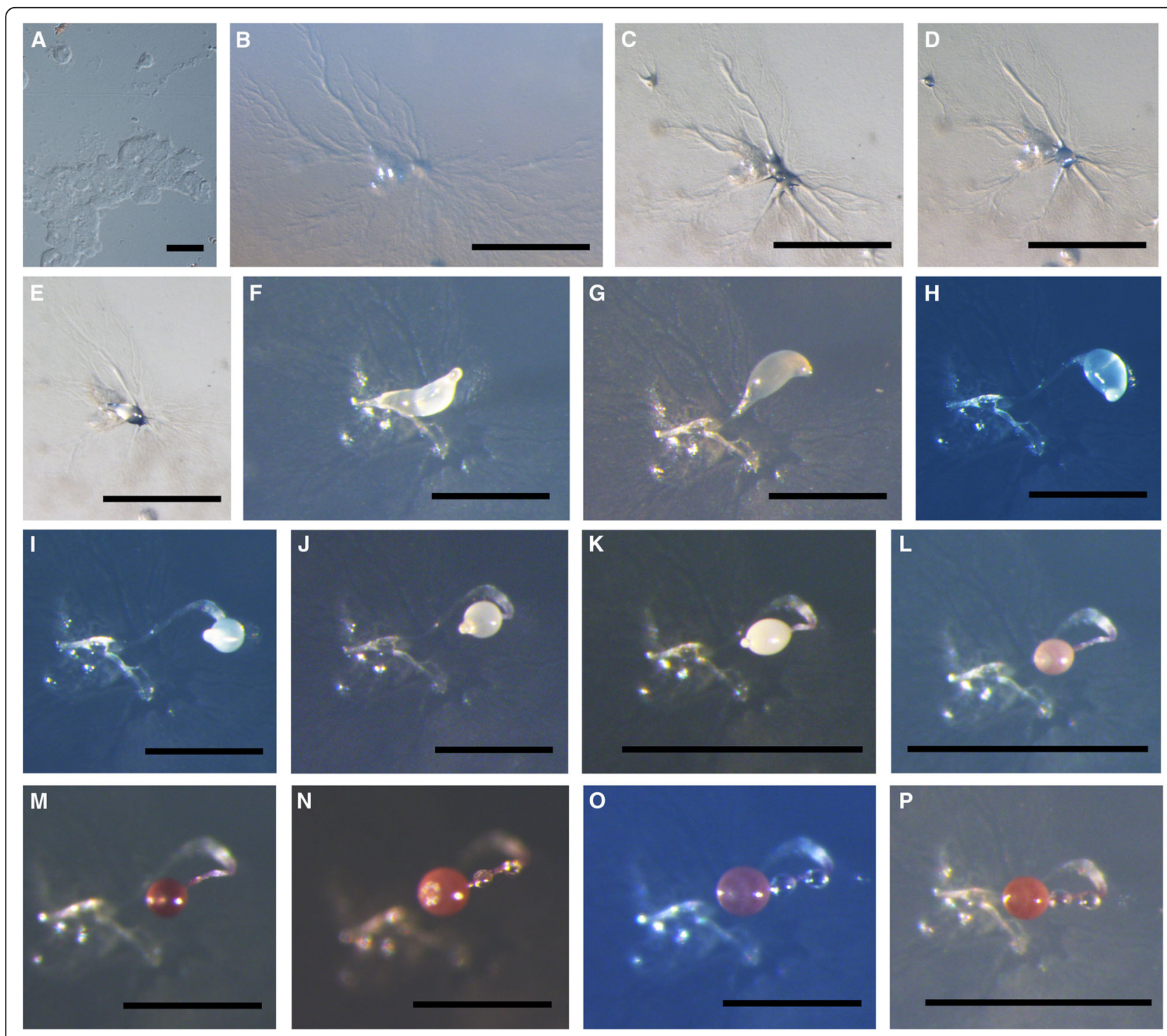

Fig. 3 Life cycle of Dictyostelium purpureum var. pseudosessile. a Myxamoebae. b-d Aggregations. e-i Formation of pseudoplasmodia. j-k Young sorocarp. I-p Fruiting of sorocarp. Scale bars: $\mathbf{a}: 20 \mu \mathrm{m}, \mathbf{b}-\mathbf{e}, \mathbf{k}, \mathbf{l}, \mathbf{p}: 1 \mathrm{~mm}, \mathbf{f}-\mathbf{j}, \mathbf{m}-\mathbf{0}: 500 \mu \mathrm{m}$ 
gradually (Fig. 3-j, k) to form globose sori at $47 \mathrm{~h}$ (Fig. 3-1), the color changes from white (Fig. 3-j), pale yellow (Fig. 3-k), pale violet (Fig. 3-l), to violet (Fig. 3-m). Forty-eight $\mathrm{h}$ after inoculating the spores on the agar, colorless sessile droplets of water begin to form on the sorophore, these are oblong (Fig. 3-n) at first but gradually change from oblong to globose (Fig. $3-0$ ), and the sorocarps finally fruit at $50 \mathrm{~h}$ (Fig. 3-p). Sorocarps are solitary, erect or semi-erect and lack of branches.

\section{Discussion}

Morphologically, Dictyostelium purpureum var. pseudosessile is a variant of $D$. purpureum Olive characterized particularly by its colorless sessile droplets of water (Fig. 4) borne at intervals along the upright sorophores and simple recurved sorophore bases. Although D. purpureum var. pseudosessile forms a clade with $D$. purpureum (Fig. 1), D. purpureum $[5,11]$ has sorophores with basal disks, whereas the new variant somewhat widens at the midpoint of sorophore but no basal disks. In order to discriminate $D$. purpureum var. pseudosessile with $D$. purpureum deeply, we mixed these strains on one agar plate according to the method of Sathe et al. [13], we found the aggregations and pesudoplasmodia have different tips, then finally form both sorocarps of $D$. purpureum var. pseudosessile and D. purpureum on one plate (Additional file 1: Fig. S1). The SSU sequences of $D$. purpureum var. pseudosessile and $D$. purpureum have 97\% identity (Additional file 1: Fig. S2). From the observations of the life cycle of $D$. purpureum, no sessile droplets of water on the sorophore were found during this process (Additional file 1: Fig. S3). Therefore, $D$. purpureum var. pseudosessile is different with $D$. purpureum in both morphology and phylogeny.

Dictyostelium purpureum var. pseudosessile is rare but is known to occur in two regions of tropical China. The morphological and molecular features of D. purpureum var. pseudosessile are considerably different compared to D. purpureum mentioned above, with the most prominent features of the new variant being the prostrate sorophore, widens at the midpoint of sorophore and simple recurved sorophore bases. The entire life cycle of $D$. purpureum var. pseudosessile extends over $3 \mathrm{~d}(50 \mathrm{~h})$. The spores germinated and released myxamoebae, and aggregations formed $32.5 \mathrm{~h}$ after inoculation. Pseudoplasmodium formation required $42 \mathrm{~h}$. Following this, the sorophores and sori begin to grow in an orderly manner and finally formed fruiting sorocarps after $50 \mathrm{~h}$.

The most important contribution of this paper is that it extends our knowledge of the distribution and ecology of dictyostelids in a region of the world where these organisms remain understudied. It seems likely that additional sampling in tropical areas of China will yield other species new to science or already described species which are not yet known to occur in China.

\section{Conclusions}

Reported herein are two isolates, each from a different area of tropical China, which represented a new variant of Dictyostelium purpureum. Our discovery of this new variant (D. purpureum var. pseudosessile) from tropical areas of China expands the biogeographical range of dictyostelids. Molecular phylogenetic analyses of the two isolates revealed that they do not belong to any previously described species. We propose that the two new isolates belong to the same species, which is characterized by violet sori, widens at the midpoint of sorophore and simple recurved sorophore bases. This new variant required $50 \mathrm{~h}$ to complete its entire life cycle, incuding spore germination, aggregation formation, pseudoplasmodium formation, and sorocarp formation.

\section{Methods}

\section{Study site ecology}

Soil samples used for the isolation of dictyostelids were collected in two tropical areas of China in January 2015. These were the Xishuangbanna Tropical Botanical Garden of the Chinese Academy of Sciences located in Yunnan Province and Qixianling Hot Springs National Forestry Park located in Hainan Province. The Xishuangbanna Tropical Botanical Garden is situated in the southwestern portion of China, whereas the Qixianling Hot Springs National Forestry Park occurs in the southernmost portion of China. Both of these two localities are located south of the Tropic of Cancer and are characterized by a tropical monsoon climate, with an annual temperatures of 21.4 $\mathrm{C}$ and 23.0 C, respectively.

\section{Sampling}

Samples, each consisting of approximately $30-50 \mathrm{~g}$ of material, were collected and placed in sterile whirl-pack plastic bags. In most instances, at least five samples were collected from each vegetation type at each locality. Afterwards, these samples were returned to the laboratory as soon as possible, following the recommendations of Cavender and Raper [3]. Each sample bag was numbered and the sample itself preserved at $4 \mathrm{C}$ in the herbarium of the Mycological Institute of Jilin Agricultural University (HMJAU), Changchun, China.

\section{Isolation and cultivation}

The isolation methods used followed those described by Cavender and Raper [3]. Each sample was weighed and diluted for an initial dilution of $1: 10$ by adding $\mathrm{ddH}_{2} \mathrm{O}$. This solution was shaken to disperse the 

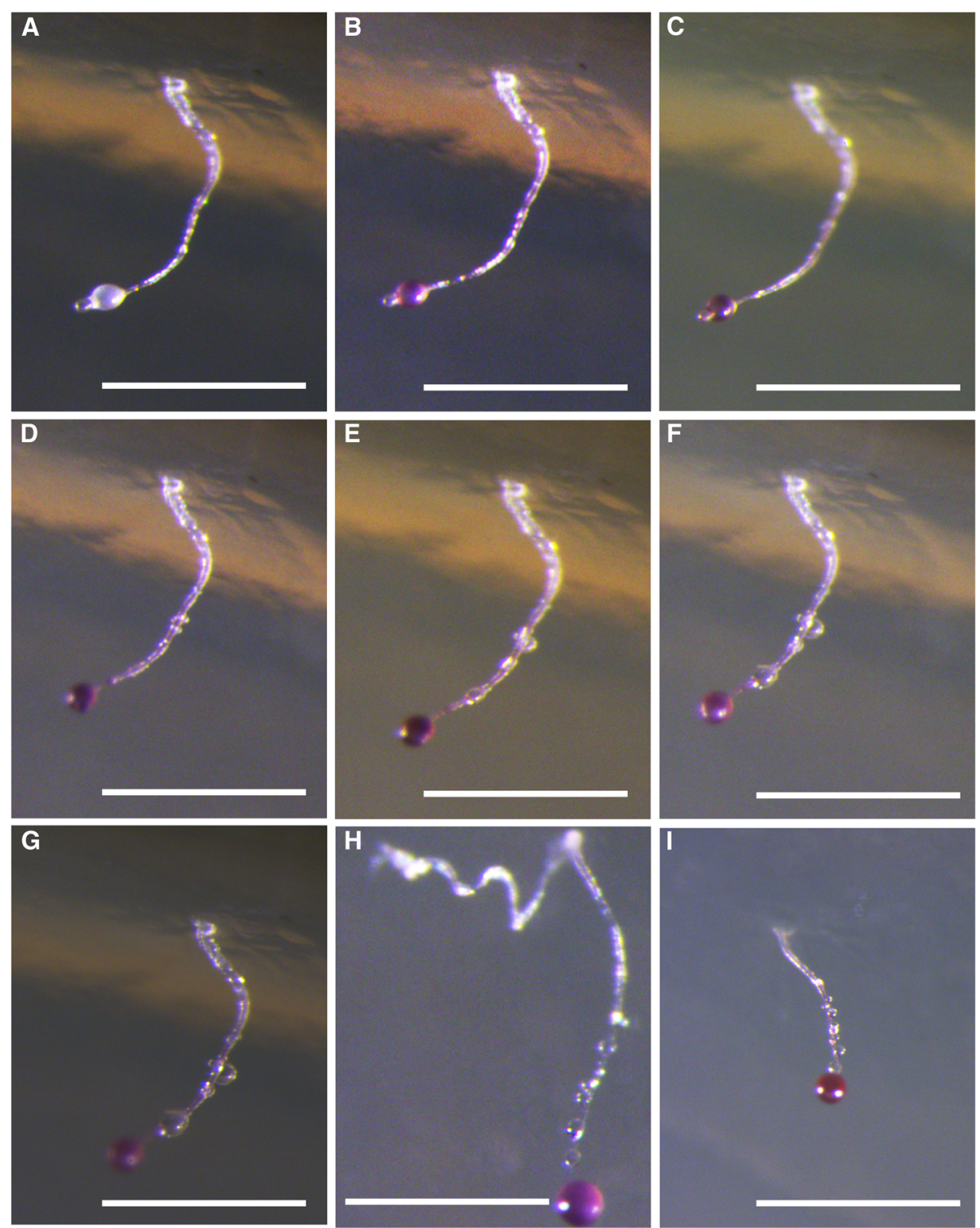

Fig. 4 Dictyostelium purpureum var. pseudosessile. $\mathbf{a}-\mathbf{g}$ Fruiting process of sorocarps, each from them were photographed in one hour. $\mathbf{h}, \mathbf{i}$ Other sorocarps. Scale bars: $\mathbf{a}-\mathbf{g}: 500 \mu \mathrm{m}, \mathbf{h}$, i: $1 \mathrm{~mm}$

material and to suspend the amoebae and spores of dictyostelids present. Afterwards, a $0.5 \mathrm{~mL}$ aliquot of the solution was added to each of five duplicate culture plates prepared with hay infusion agar [5]. Approximately $0.4 \mathrm{~mL}$ of a heavy suspension of the bacterium Escherichia coli was added to each culture plate as a food source. The plates were incubated at a temperature of $23 \mathrm{C}$, with a $12 \mathrm{~h}$ light and dark cycle. Each plate was examined at least once a day for two weeks after the appearance of initial aggregations. Each isolate was purified and cultivated for taxonomic studies and preservation on non-nutrient water agar plates with $E$. coli pregrown for $12-24 \mathrm{~h}$. Spores from these plates were frozen in HL 5 media [14] and stored at $-80 \mathrm{C}$ in HMJAU.

\section{Morphological features and life cycle observations}

Dictyostelid isolates were identified with the use of the descriptions provided by Raper [5], whose nomenclature also was followed except for those species recently assigned to new genera in the system of classification proposed by Sheikh et al. [11]. In the primary isolation plates, the location of each early aggregating clone and developing sorocarp was marked. The characteristic stages in the life cycle, including cell aggregation and the formation of pseudoplasmodia and ultimately sorocarps, 
were observed under a Zeiss dissecting microscope (Axio Zoom V16) with a $1.5 \times$ objective and a $10 \times$ ocular. Slides of sorocarps were prepared with water as the mounting medium. Characteristics of spores, sorophores, and sorocarps were observed and measured on the slides by using a Zeiss light microscope (Axio Imager

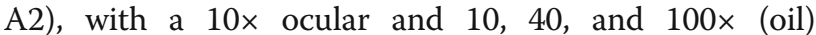

objectives. Photographs were taken with a Zeiss Axiocam 506 color microscope camera.

Observation of spore germination. Hanging drop cultures, as described by Keller and Schoknecht [15], were prepared for observation of spore germination. Spores obtained from a sorus were mixed with a droplet of sterile water on the undersurface of a $22-\mathrm{mm}$ square cover

Table 1 Species of dictyostelids included in the present study. Information is provided on the samples considered and the GenBank accession number for SSU sequences

\begin{tabular}{|c|c|c|}
\hline Species & Voucher specimen & GenBank accession number (SSU) \\
\hline Acytostelium amazonicum Cavender \& Vadell & HN1B1 & HQ141511.1 \\
\hline A. leptosomum Raper & FG12 & AM168111.1 \\
\hline A. serpentarium Cavender et al. & SAB3A & AM168113.1 \\
\hline A. subglobosum Cavender & LB1 & AM168110.1 \\
\hline Cavenderia delicata (H. Hagiw.) S. Baldauf, S. Sheikh \& Thulin & TNS-C-226 & AM168093.1 \\
\hline C. fasciculata (F. Traub et al.) S. Baldauf, S. Sheikh \& Thulin & $\mathrm{SH} 3$ & AM168087.1 \\
\hline C. myxobasis (Cavender et al.) S. Baldauf, S. Sheikh \& Thulin & NT2A & HQ141522.1 \\
\hline Coremiostelium polycephalum (Raper) S. Baldauf, S. Sheikh, Thulin \& Spiegel & MY1-1 & AM168056.1 \\
\hline C. polycephalum & Landolt \#1130 SS3B & HQ141488.1 \\
\hline Dictyostelium brunneum Kawabe & WS700 & AM168031.1 \\
\hline D. crassicaule H.Hagiw. & $93 \mathrm{HO}-33$ & AM168037.1 \\
\hline D. purpureum Olive & C143 & AM168060.1 \\
\hline D. purpureum var. pseudosessile Li Y, P Liu, Y Zou SL Stephenson et JG Hou & 4446 & $\mathrm{MH} 280023^{\mathrm{a}}$ \\
\hline D. purpureum var. pseudosessile & 4637 & $\mathrm{MH} 280022^{\mathrm{a}}$ \\
\hline D. pseudobrefeldianum H.Hagiw. & $91 \mathrm{HO}-8$ & AM168059.1 \\
\hline D. robustum H.Hagiw. & TNS-C-219 & AM168064.1 \\
\hline D. rosarium Raper \& Cavender & M45 & AM168065.1 \\
\hline D. septentrionale Cavender & AK2 & AM168067.1 \\
\hline Hagiwaraea coeruleostipes (Raper \& Fennell) S. Baldauf, S. Sheikh \& Thulin & CRLC53B & AM168036.1 \\
\hline H. radiculata (Cavender et al.) S. Baldauf, S. Sheikh \& Thulin & ML5A & HQ141494.1 \\
\hline H. vinaceofusca (Raper \& Fennell) S. Baldauf, S. Sheikh \& Thulin & CC4 & AM168062.1 \\
\hline Heterostelium anisocaule (Cavender et al.) S. Baldauf, S. Sheikh \& Thulin & NZ47B & AM168096.1 \\
\hline H. colligatum (Vadell \& Cavender) S. Baldauf, S. Sheikh \& Thulin & $\mathrm{HN} 13 \mathrm{C} 1$ & HQ141505.1 \\
\hline H. filamentosum (F.Traub et al.) S. Baldauf, S.Sheikh \& Thulin & SU-1 & AM168100.1 \\
\hline H. oculare (Cavender et al.) S. Baldauf, S. Sheikh \& Thulin & - & HQ141497.1 \\
\hline Polysphondylium laterosorum (Cavender) S. Baldauf, S. Sheikh \& Thulin & AE4 & AM168046.1 \\
\hline P. violaceum Bref. & 209 & HQ141486.1 \\
\hline Raperostelium gracile (H.Hagiw.) S. Baldauf, S. Sheikh \& Thulin & TNS-C-183 & AM168078.1 \\
\hline R. monochasioides (H.Hagiw.) S. Baldauf, S. Sheikh \& Thulin & HAG653 & AM168052.1 \\
\hline R. potamoides (Cavender et al.) S. Baldauf, S. Sheikh \& Thulin & FP1A & AM168069.1 \\
\hline R. tenue (Cavender et al.) S. Baldauf, S. Sheikh \& Thulin & PJ6 & AM168094.1 \\
\hline Rostrostelium ellipticum (Cavender) S. Baldauf, S. Sheikh \& Thulin & AE2 & AM168112.1 \\
\hline Synstelium polycarpum (F.Traub et al.) S. Baldauf, S. Sheikh \& Thulin & VE1b & AM168057.1 \\
\hline S. polycarpum & OhioWILDS & AM168058.1 \\
\hline Tieghemostelium lacteum (Tiegh.) S. Baldauf, S. Sheikh \& Thulin & - & AM168045.1 \\
\hline T. menorah (Vadell \& Cavender) S. Baldauf, S. Sheikh \& Thulin & M1 & AM168073.1 \\
\hline
\end{tabular}

${ }^{a}$ samples and sequences obtained in the present study 
glass. The cover glass was then inverted over a depression slide. Vaseline was used to ring the edges of the cover glass. Spores were freely suspended in the water droplet. Characteristics of the myxamoebae were observed and photographed with a Zeiss light microscope (Axio Imager A2), using the 10x ocular and 10, 40, and $100 \times$ (oil) objectives.

\section{Nomenclature}

According to the International Code of Nomenclature used for algae, fungi, and plants, the electronic version of this article in Portable Document Format (PDF) will represent a published work. In addition, new names contained in this study have been submitted to MycoBank and each will be allocated a unique MycoBank number which will be accessible through MycoBank, Index Fungorum, GBIF and other international biodiversity initiatives, where they will be made available to the Global Names Index.

\section{DNA isolation, PCR amplification and sequencing}

After amoebae had cleared E. coli on the water agar media, the spores of the particular dictyostelid isolate being studied were collected with a sterile tip, mixed with the lysed buffer of the MiniBEST Universal Genomic DNA Extraction Kit Ver.5.0 (Takara, Japan) following the manufacturer's protocol. The genomic DNA was used directly for the $18 \mathrm{~S}$ PCR amplification using the primers 18SF-A (AACCTGGTTGATCCTGCCAG) and 18SR-B (TGATCCTTCTGCAGGTTCAC) [16] along with D542F (ACAATTGGAGGGCAAGTCTG3) and D1340R (TCGAGGTCTCGTCCGTTATC) [9]. PCR products were sent to Sangon Biotech Co., Ltd. (Shanghai, China) for sequencing. Sequences obtained were deposited at GenBank database. The isolates and the NCBI GenBank accession numbers of SSU DNA sequences used in present study are listed in Table 1 .

\section{Phylogenetic analysis}

The newly-generated sequences were checked and then submitted to GenBank. The ITS and SSU sequences were aligned and compared separately using the program MUSCLE v.3.6 [17, 18] and then manually adjusted in MEGA 7.0 [19]. Maximum likelihood (ML) analyses were performed using RAxML v7 [20]. In the ML analyses, the best-fit substitution models were estimated using GTR submission model and a gamma correction for rate variation among sites (GTRGAMMA), using the CIPRES server. The statistical support of clades was assessed with 1000 rapid-bootstrap (BS) replications.

\section{Additional file}

Additional file 1: Fig. S1. Mixed culture of Dictyostelium purpureum var. pseudosessile with D. purpureum. A-C Aggregations. D Pseudoplasmodia. F Sorocarps. Scale bars: A,B,D: 1 mm, C: $500 \mu m, E: 2$ mm. Fig. S2. Blast of SSU sequences of Dictyostelium purpureum var. pseudosessile with D. purpureum which have $97 \%$ identity. Fig. S3. Life cycle of Dictyostelium purpureum. The time of each stage showed on the top right corner. A-D Aggregations. E,F Pseudoplasmodia. G-L Sorocarps. Scale bars: A-H: 2 mm, I-L: 1 mm. (DOCX 344 kb)

\section{Acknowledgements}

We wish to express our appreciation to the editor and the reviewers for their comments and valuable reviews of this manuscript.

\section{Funding}

This research was supported by the National Natural Science Foundation of China (No. 31870015, 31300016), the Science and Technology Development Program of Jilin Province (No. 20180101273JC), the Program of Creation and Utilization of Germplasm of Mushroom Crop of the "111" Project (No. D17014), and the Science and Technology Research Programs of the Education Department of Jilin Province in the Thirteenth Five-Year Plan (No. JJKH20180671KJ).

Availability of data and materials

Sequence data are available in GenBank. The nomenclature of the new variant in the present study is available in MycoBank.

\section{Authors' contributions}

YZ, PL and JH carried out the research, PL wrote the manuscript, YL designed the research, SLS revised the manuscript. All authors read and approved the final version of the manuscript.

Ethics approval and consent to participate

Not applicable.

Consent for publication

Not applicable.

Competing interests

The authors declare that they have no competing interests.

\section{Publisher's Note}

Springer Nature remains neutral with regard to jurisdictional claims in published maps and institutional affiliations.

\section{Author details}

${ }^{1}$ Engineering Research Center of Chinese Ministry of Education for Edible and Medicinal Fungi, Jilin Agricultural University, Changchun 130118, People's Republic of China. ${ }^{2}$ Department of Biological Sciences, University of Arkansas, Fayetteville, AR 72701, USA.

Received: 9 May 2018 Accepted: 4 March 2019

Published online: 14 March 2019

\section{References}

1. Singh BN. Studies on soil Acrasieae. 1. Distribution of species of Dictyostelium in soils of Great Britain and the effects of bacteria on their development. J. Gen. Microbiol. 1947. https://doi.org/10.1099/00221287-1-111.

2. Cavender JC, Raper KB. The Acrasieae in nature. I. Isolation. Am J Bot. 1965. https://doi.org/10.2307/2439943.

3. Cavender JC, Raper KB. The Acrasieae in nature. II. Forest soil as a primary habitat. Am J Bot. 1965b. https://doi.org/10.2307/2439944.

4. Raper KB. Acrasiomycetes. In: Ainsworth GC, Sparrow FK, Sussman AS, editors. The Fungi. United States: Academic Press; 1973. p. 9-36.

5. Raper KB. The Dictyostelids. New Jersey: Princeton University Press, Princeton; 1984. p. 1-453 
6. Fets L, Kay R, Velazquez F. Dictyostelium Current Biology. 2010. https://doi. org/10.1016/j.cub.2010.09.051.

7. Brefeld O. Dictyostelium mucoroides. Ein neuer Organismus aus der Verwandschaft der Myxomyceten. Abh Seckenburg Naturforsch Ges. 1869;7: 85-107.

8. Kirk PM, Cannon PF, Minter DW, Stalpers JA. Dictionary of the fungi. 10th ed: CABl; 2008. p. 1-640.

9. Schaap P, Winckler T, Nelson M, Alvarez-Curto E, Elgie B, Hagiwara H, Cavender JC, Milano-Curto A, Rozen DE, Dingermann T, Mutzel R, Baldauf SL. Molecular phylogeny and evolution of morphology in the social amoebas. Science. 2006. https://doi.org/10.1126/science.1130670.

10. Romeralo M, Cavender JC, Landolt JC, Stephenson SL, Baldauf SL. An expanded phylogeny of social amoebas (Dictyostelia) shows increasing diversity and new morphological patterns. BMC Evol Biol. 2011. https://doi. org/10.1186/1471-2148-11-84

11. Sheikh S, Thulin M, Cavender JC, Hernández RE, Kawakami SI, Lado C, Landolt JC, Nanjundiah V, Queller D, Shaap P, Strassmann J, Spiegel F, Stephenson SL, Vadell EM, Baldauf SL. A new classification of the dictyostelids. Protist. 2018. https://doi.org/10.1016/.jprotis.2017.11.001.

12. Liu P, Li Y. New records of dictyostelids from China. Nova Hedwigia. 2012. https://doi.org/10.1127/0029-5035/2012/0010.

13. Sathe $\mathrm{S}$, Khetan $\mathrm{N}$, Nanjundiah V. Interspecies and intraspecies interactions in social amoebae. J Evol Biol. 2014. https://doi.org/10.1111/jeb.12298.

14. Cocucci SM, Sussman M. RNA in cytoplasmic and nuclear fractions of cellular slime mold amebas. J Cell Biol. 1970. https://doi.org/10.1083/jcb.45.2. 399

15. Keller HW, Schoknecht JD. Life cycle of a new annulate-spored species of Didymium. Mycologia. 1989. https://doi.org/10.2307/3759707.

16. Medlin L, Elwood HJ, Stickel S, Sogin M. The characterization of enzymatically amplified eukaryotic 16S-like rRNA-coding regions. Gene. 1988. https://doi.org/10.1016/0378-1119(88)90066-2.

17. Edgar RC. MUSCLE: multiple sequence alignment with high accuracy and high throughput. Nucleic Acids Res. 2004. https://doi.org/10.1093/nar/ gkh340.

18. Edgar RC. MUSCLE: a multiple sequence alignment method with reduced time and space complexity. BMC Bioinformatics. 2004. https://doi.org/10. 1186/1471-2105-5-113

19. Kumar S, Stecher G, Tamura K. MEGA7: Molecular Evolutionary Genetics Analysis Version 7.0 for Bigger Datasets. Molecular Biology \& Evolution. 2016; https://doi.org/10.1093/molbev/msw054.

20. Stamatakis A. RAxML-VI-HPC: maximum likelihood-based phylogenetic analyses with thousands of taxa and mixed models. Bioinformatics. 2006 https://doi.org/10.1093/bioinformatics/btl446.

Ready to submit your research? Choose BMC and benefit from:

- fast, convenient online submission

- thorough peer review by experienced researchers in your field

- rapid publication on acceptance

- support for research data, including large and complex data types

- gold Open Access which fosters wider collaboration and increased citations

- maximum visibility for your research: over $100 \mathrm{M}$ website views per year

At $\mathrm{BMC}$, research is always in progress.

Learn more biomedcentral.com/submissions 